\title{
Fission yields and cross sections: correlated or not?
}

\author{
Dimitri Alexandre Rochman ${ }^{1, *}$ and Eric Bauge ${ }^{2,3}$ \\ ${ }^{1}$ Reactor Physics and Thermal hydraulic Laboratory, Paul Scherrer Institut, Villigen, Switzerland \\ ${ }^{2}$ CEA DAM DIF, 91297 Arpajon, France \\ ${ }^{3}$ Université Paris Saclay, CEA, Laboratoire Matière sous Conditions Extrêmes, 91680 Bruyères-le-Châtel, France
}

Received: 14 October 2020 / Received in final form: 8 February 2021 / Accepted: 18 February 2021

\begin{abstract}
Cross sections and fission yields can be correlated, depending on the selection of integral experimental data. To support this statement, this work presents the use of experimental isotopic compositions (both for actinides and fission products) from a sample irradiated in a reactor, to construct correlations between various cross sections and fission yields. This study is therefore complementing previous analysis demonstrating that different types of nuclear data can be correlated, based on experimental integral data.
\end{abstract}

\section{Introduction}

During the last decades, the importance of accurately estimating a degree of confidence in nuclear simulations has gathered an increasing amount of attention. Whereas measured values were historically often followed by uncertainties, calculated quantities were for too long provided alone. Nowadays, due to safety and economical pressures, increased computer power, and needs of informed decision making, it has become more common that uncertainties go along with best estimations. Nuclear data is no exception to this trend, and their recommended values (also called evaluations) now comes with a fair amount of covariance matrices in the latest national and international efforts [1-4].

Traditionally, such covariance matrices were first provided to the user community for neutron-induced reactions and their cross sections (called XS in the following), as being often the most sensitive quantities for many nuclear applications. At equal level of importance is the average number of neutrons emitted per fission $(\nu)$, which nowadays also comes with its own set of covariance matrices, and often the emitted spectra and angular distributions as well. Frequently, these matrices link different energy regions of standard deviations of the same quantity, and can also be provided for different reactions. But, they are restricted to the same quantities for a given isotope: for instance, for the energy dependence of the fission cross section of ${ }^{235} \mathrm{U}$, or for the energy dependence between its capture and fission cross sections. Nevertheless, in modern evaluations, these correlations are not yet provided between uncertainties of different quantities, such as XS

\footnotetext{
*e-mail: dimitri-alexandre.rochman@psi.ch
}

and $\nu$, or between isotopes. The reason mostly lies in the difficulty to construct such correlations based on selected differential measurements and reaction calculations. There is a noticeable exception, as the vast majority of measurements are performed relatively to a standard cross section (such as ${ }^{197} \mathrm{Au}(\mathrm{n}, \gamma)$, or ${ }^{235} \mathrm{U}(\mathrm{n}, \mathrm{f})$ ); therefore almost all evaluated nuclear data must be correlated to some of these standards, and therefore also between themselves [5]. But this is generally not yet considered in the evaluation efforts for the recommended uncertainties and correlations (from a practical aspect, the evaluation format was not defined to accommodate for such various correlations; an update of such format would therefore be necessary).

Outside the major evaluated libraries, it was recently shown that it is possible to correlate different quantities, for instance XS and $\nu$, and even different isotopes (e.g. ${ }^{235} \mathrm{U}$ and ${ }^{238} \mathrm{U}$ ) using so-called integral experimental data. Such experiments, based on various isotopes and complex geometries, or providing measured quantities sensitive to many reactions, allow to construct a vast amount of new correlations when using Bayesian methods such as the so-called BMC (Bayesian Monte Carlo), BFMC (Backward-Forward Monte Carlo), or MOCABA [6-9] (other methods as presented in Refs. [10-20] could also be used, even if developed towards different goals). These methods allow to take into account integral data during an evaluation process, based on an adjustment of specific quantities. The experimental integral data used for these studies were mostly criticality benchmarks, with the provided $k_{\text {eff }}$ or reaction rates, but also reactor data such as boron concentrations [21-29]. It was also shown that still using differential data with oxide targets, it is possible to provide correlations between oxygen, hydrogen and the other compound isotopes [30]. 
These various new results indicate that it is possible to build covariance matrices for cross sections between isotopes, or between $\nu$, XS and fission spectra for a specific isotopes, or finally between XS of different isotopes. A larger number of reactions, quantities and isotopes could therefore be considered as correlated. The question of recommending such correlation matrices in evaluations is still debated among specialists.

Following this line of research, some quantities have still been left aside from this global correlation effort, namely fission yields. In the following, the term "fission yield" will designate without distinction independent or cumulative fission yields (called FY). It is a known deficiency that current evaluated libraries do not provide correlation matrices for fission yields, neither for a specific fissioning isotope (such as all fission yields from ${ }^{235} \mathrm{U}(\mathrm{n}, \mathrm{f})$ ), or between fissioning isotopes. This represents a practical problem for nuclear data users, as uncertainties on quantities dependent of FY (e.g. long-lived fission products from spent fuel) are believed to be too large, due to the lack of FY correlations [31-34]. As mentioned before, the current evaluation format is not defined to propose such correlations for FY; there are nevertheless efforts to update such format.

To help resolving this problem of missing correlations, the present work demonstrates that specific integral data can be used to construct correlations between fission yields, but also between XS and FY. Such correlations were not yet put into lights and this paper presents an example of XS-FY correlations using measured and simulated isotopic concentrations from a specific sample irradiated in various reactor cycles. In the following, we first describe the considered experimental data and simulation tools (sample, irradiation, measurements), the method used to build such correlations (BFMC) and finally the results. It will hopefully be clear that fission yields and cross sections can be correlated, thus extending again the dimension of correlated nuclear data.

\section{On the use of a PIE sample}

As presented in the introduction, many types of nuclear data correlations can be constructed using integral data as constraints. Most common integral data used during the evaluation process are the criticality state of a system (or criticality benchmark) [35], called $\mathrm{k}_{\text {eff }}$, sometimes accompanied by spectral indexes (ratios of capture over fission reaction rates) or reaction rates for capture, $(n, 2 n),(n, p)$ or $(\mathrm{n}, \alpha)$ reactions in more complex systems [36,37].

In the present work, we are proposing to take advantage of the so-called Post Irradiation Examination samples (or PIE samples), which consist in the destructive analysis of part of an irradiated fuel assembly. Such analysis provides measurements for the isotopic concentrations of specific isotopes (in $\mathrm{mg} / \mathrm{gU}$, for actinides and fission products) which live long enough to be measured a few years after the last assembly irradiation. Typically, such PIE data are used in the validation of transport and depletion codes (as presented for instance in Refs. [38-40]), but can also be used in nuclear data-related activities. Such samples are made of $\mathrm{UO}_{2}$ or $\mathrm{MOX}$ fuel, are irradiated in conventional water reactors for various cycles, and can reach burnup values of 50 to $70 \mathrm{MWd} / \mathrm{kgU}$ (and sometimes more). Therefore, a number of minor actinides can be measured (up to ${ }^{245} \mathrm{Cm}$ ) with a number of fission products, from the most produced during fission (e.g. ${ }^{99} \mathrm{Tc}$ ) to the less produced (e.g. Gd). Additionally, the neutron energy of relevance is mostly in the thermal region.

The irradiation and cooling of such samples can be simulated with a variety of codes, and it was demonstrated that nuclear data, both cross sections and fission yields, can greatly influence isotopic concentrations [39]: if the concentration of actinides mainly depends on capture and fission cross sections, fission yields can be the most influential parameters for specific fission products (such as Cs isotopes). PIE samples then become very suited integral experiments to correlate different cross sections themselves, as well as cross sections and fission yields.

Among available PIE samples, the ARIANE GU1 sample is selected in this work. It is part of the open-literature database SFCOMPO [41,42] and consists of a $\mathrm{UO}_{2}$ sample, enriched at $3.5 \%$ in ${ }^{235} \mathrm{U}$, and was irradiated during 4 consecutive cycles in the Gösgen power plant (approximately 3.5 years of irradiation, followed by 6 years cooling time). The sample burnup is estimated to be close to 58.6 $\mathrm{MWd} / \mathrm{kgU}$, and a number of isotopic concentrations are reported (details can be found in the ARIANE report). The consideration of a unique experiment is certainly not satisfactory from a comprehensive perspective. The correlations extracted in the following are therefore solely presented for illustration; in a more realistic evaluation work, a larger number of experiments needs to be selected, to be representative enough of the current experimental knowledge. For readers interested in details and agreements between calculated and measured isotopic contents for the GU1 sample, we refer to the study presented in reference [43]

\section{Method to build correlations}

Before defining the method applied to construct correlations, the definition of the correlation itself in the context of this paper can be useful. Mathematical definitions of the correlation coefficient can be found in references $[27,43]$, and for the present work, its interpretation will be the following. A correlation coefficient $\rho$ between two quantities $A$ and $B$ indicates that if $A$ is changing by $1 \times \sigma_{A}$ ( $\sigma_{A}$ being the standard deviation of $A$ ), then $B$ will be changing by $\rho \times \sigma_{B} . \rho$ can be positive or negative, and varies between -1 and 1 . In the following, if one cross section $A$ (at a given energy) is positively correlated with another one $B$, it means that if $A$ increases, $B$ also increases. In the case of negative correlation, $A$ and $B$ vary in opposite directions. This definition is useful to understand the following representative case: if a measured quantity $E$ is created by the $A$ process and disappears because of the $B$ process, keeping $E$ constant implies that $A$ and $B$ vary in same direction; $A$ and $B$ therefore become positively correlated. 
If $E$ is created by both $A$ and $B$ processes, then keeping $E$ constant implies that $A$ and $B$ become negatively correlated. As presented in the following, posterior correlations (created by taking into account a specific measured $E$ value) can be understood with these simple steps in mind, as Bayesian methods such as BFMC tend to produce posterior calculated quantities closer to experimental data.

The simulation of such sample is presented in reference [43] and the calculated results (nominal and perturbed) of this reference are used in the present work. In short, a model with the deterministic code CASMO5 is used for simulating the irradiation and cooling times [44], and the isotopic concentrations of a number of isotopes are calculated. It allows to calculate simple $C / E$ ratios for each isotope (Calculation over Experimental values). Additionally, a number of nuclear data can be varied, and for each perturbed set of cross sections and fission yields, new $C$ values are obtained, leading to calculated uncertainties $\Delta C$, due to nuclear data.

In these calculations, the nominal values are based on the ENDF/B-VII.1 library; the cross section covariance matrices are also obtained from the ENDF/B-VIII.0 library, whereas in the case of fission yields, an in-house tool is used for prior correlations between some yields (all uncertainties also come from ENDF/B-VIII.0) [45], mainly because of the lack of correlation in all evaluated libraries. The covariance information from the ENDF/B-VIII.0 library are therefore associated with the best-estimate XS from the ENDF/B-VII.1 library. While this is theoretically not a recommended practice, this approach accommodates for pragmatic approach due to limited accessibility to CASMO5 libraries at the time of this study. The varied nuclear data (all perturbed together) are the following:

- capture cross sections: ${ }^{103} \mathrm{Rh},{ }^{109} \mathrm{Ag},{ }^{133} \mathrm{Cs}$, ${ }^{143,145} \mathrm{Nd},{ }^{147} \mathrm{Pm},{ }^{149,151,152} \mathrm{Sm},{ }^{155} \mathrm{Eu},{ }^{235,238} \mathrm{U}$, ${ }^{237} \mathrm{~Np},{ }^{239-242} \mathrm{Pu},{ }^{241} \mathrm{Am},{ }^{242-244} \mathrm{Cm}$,

- fission cross sections: ${ }^{235,238} \mathrm{U},{ }^{237} \mathrm{~Np},{ }^{239-242} \mathrm{Pu}$, ${ }^{241} \mathrm{Am},{ }^{242-244} \mathrm{Cm}$,

- fission yields for thermal neutron-induced fission: ${ }^{235} \mathrm{U},{ }^{239,241} \mathrm{Pu}$,

- fission yields for fast neutron-induced fission: ${ }^{238} \mathrm{U}$.

All cross section covariance matrices are provided in 19 energy groups, from 0 to $20 \mathrm{MeV}$, as indicated in reference [39]. In total, more than 4000 CASMO5 simulations are performed with the above perturbed nuclear data. In reference [43], it was demonstrated that other nuclear data do no significantly affect the isotopic concentrations of interest.

The method used to calculate correlations between different nuclear data is the BFMC method. It was presented in reference [6] and applied in some of the previous citations. A short summary is provided here. For each of the random calculations $i$ based on perturbed nuclear data, calculated isotopic concentrations are obtained. For a specific isotope (for instance ${ }^{99} \mathrm{Tc}$ ), the calculated values $C_{i}$ ( $i$ varying from 1 to 4000) can be compared with the measured value $E$. The comparison can be used to calculate a weight $w_{i}$, representing the degree of agreement between both quantities:

$$
w_{i}=\exp \left[-\left(\frac{C_{i} / E}{\left(C_{j} / E\right)_{\min }}\right)^{2}\right] .
$$

In this equation, the ratio with the "min" label corresponds to the case $j$ leading to the best agreement between the calculated and experimental value (ideally, $C_{j}$ and $E$ are equal). The use of this normalization factor is the main difference between the BMC and BFMC method: it helps to compensate for the so-called "model defects" that the CASMO5 simulations contain.

The use of weights $w_{i}$ favors the samples which agree with the experiment with the effect that the posterior distribution is in better agreement with $E$, compared to the prior value. They are then used to calculate posterior distributions, for instance using weighted averages, weighted standard deviations, or weighted correlations. Equations can for instance be found in reference [26]. In the following, only the prior and posterior correlation values will be presented, but such Bayesian approach also provides posterior central values and variances.

Equation (1) could be generalized to the use of many experimental data at once in the calculation of weights (ideally coming both integral and differential experiments), but in the following, experimental data will only be considered one by one to understand the origin of the constructed correlations.

As mentioned, the irradiation of the GU1 sample is simulated and the reported experimental values $\mathrm{E}$ from the ARIANE report are used [41]. 50 isotopes were measured (18 actinides, 32 fission products), but only a limited number of them present an acceptable $C / E$ ratio: only $21 C / E$ cases among 50 are within a $1 \sigma$, with $\sigma$ being $\sqrt{(\Delta E)^{2}+(\Delta C)^{2}}$. This is not uncommon in PIE validation work, for instance due to approximations in the simulations, (very) small experimental uncertainties, or partial isotopic dissolution. Therefore, only a limited number of isotopic calculations will be used in the following for equation (1).

In the next section, specific representative results will be presented and detailed, and to simplify the interpretation of the correlations, only single isotopic composition will be analyzed at once.

\section{Results}

Different cases are presented in the following. First, only the constructed correlations between cross sections (XS) are analyzed, followed by the correlations between fission yields (FY) only. Finally the mixed cases of correlations between FY and XS are discussed.

All presented figures of correlations between nuclear data follow the same structure. They are made of a number of square blocks, each block representing a specific nucleus and reaction (for instance ${ }^{235} \mathrm{U}(\mathrm{n}, \mathrm{f})$ ). They are separated by solid black lines. In the case of cross sections, such blocks are divided in ten zones (possibly filled 

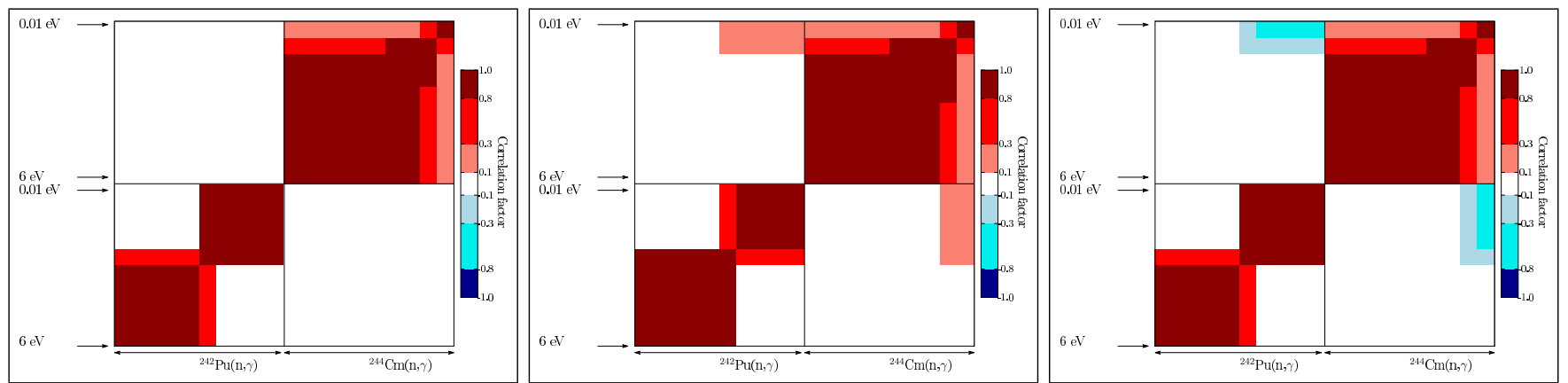

Fig. 1. Case of correlations between cross sections for ${ }^{242} \mathrm{Pu}(\mathrm{n}, \gamma)$ and ${ }^{244} \mathrm{Cm}(\mathrm{n}, \gamma)$. Left: prior correlation matrix without PIE data; middle: posterior correlation matrix built using the PIE measurement from ${ }^{244} \mathrm{Cm}$; right: posterior correlation matrix built using the PIE measurement from ${ }^{245} \mathrm{Cm}$.

with blue or red squares), each one representing one neutron incident energy group, in total ranging from $0.01 \mathrm{eV}$ to $6 \mathrm{eV}$. In the case of fission yields, each of the ten zones represents one specific fission yield. Both $x$ and $y$ axes are similar.

\subsection{Example 1: Correlations among cross sections, for the same reaction channel}

This example presents the case of the correlations between the capture cross sections of two different isotopes: ${ }^{242} \mathrm{Pu}$ and ${ }^{244} \mathrm{Cm}$. Both of these isotopes were not present in the sample at the beginning of the irradiation, and are built-up by neutron capture on ${ }^{239} \mathrm{Pu}$ (for ${ }^{242} \mathrm{Pu}$ ) and on ${ }^{242} \mathrm{Pu}$ (for ${ }^{244} \mathrm{Cm}$ ). Both of these actinides have a small fission cross section in the thermal range, so that their disappearance is mainly due to their capture cross sections. Examples of different correlations, obtained by taking into account different isotopic compositions $E$ in the BFMC weighting process are presented in Figure 1. The left matrix represents the prior correlation matrix, as obtained from the ENDF/B-VIII.0 covariance matrices. As observed, energy-energy correlations within the same reaction exist, but not between isotopes. This is a common feature of all evaluated libraries, where cross-isotope correlations are rarely provided.

The middle matrix presents the posterior correlations when using the experimental isotopic composition of ${ }^{244} \mathrm{Cm}$ as the measured value $E$. As mentioned, ${ }^{244} \mathrm{Cm}$ is mainly produced from neutron capture on ${ }^{242} \mathrm{Pu}$, and disappears due its own radiative capture. Therefore the positive posterior correlation observed between both isotopes in this matrix can typically be explained by the example of reactions $A$ and $B$ from Section 3: keeping the ${ }^{244} \mathrm{Cm}$ constant implies that both capture cross sections are positively correlated, as observed.

The left correlation matrix exhibits the opposite behavior, simply by considering the ${ }^{245} \mathrm{Cm}$ as the measured value $E$ instead of ${ }^{244} \mathrm{Cm}$. In this case, ${ }^{245} \mathrm{Cm}$ is solely produced by the ${ }^{244} \mathrm{Cm}(\mathrm{n}, \gamma)$ reaction, and indirectly also by the ${ }^{242} \mathrm{Pu}(\mathrm{n}, \gamma)$ reaction. Therefore both reactions contribute to the build-up of ${ }^{245} \mathrm{Cm}$ : to keep ${ }^{245} \mathrm{Cm}$ constant, if one increases, the other one needs to decrease. They are therefore negatively correlated.
This example illustrates that different types of correlations can be built, depending on the choice of the considered experimental value $E$. It clearly indicates that a correlation factor is only meaningful within a given context which depends on the choice of the experimental value $E$ used to calculate the weighted posterior distribution. As detailed in the discussion section, these correlations depend on the characteristics of the irradiation of the sample, and other correlations can be found for other irradiation conditions.

\subsection{Example 2: Correlations among cross sections, for different channels}

As previously indicated, correlations between specific cross sections are usually obtained and included in evaluated nuclear data libraries, based on theoretical reaction calculations and differential data. But similarly to the previous example, a number of actinide cross sections can be correlated due to specific PIE measurements and Figure 2 illustrates another of such cases. The left matrix is the prior nuclear data correlation matrix, as obtained from sampling specific reactions, based on the covariance matrices from the ENDF/B-VIII.0 library. One can observe that cross sections for four reactions are presented, namely ${ }^{235} \mathrm{U}(\mathrm{n}, \mathrm{f}),{ }^{238} \mathrm{U}(\mathrm{n}, \gamma),{ }^{239} \mathrm{Pu}(\mathrm{n}, \mathrm{f})$ and ${ }^{239} \mathrm{Pu}(\mathrm{n}, \gamma)$. Different isotopes are not correlated, but strong energyenergy correlations can appear for a specific isotope, and also between its reactions (note the positive correlations between the ${ }^{239} \mathrm{Pu}$ capture and fission cross sections). The structure of these correlations, which is not the subject of this paper, comes mainly from the physics of resonances in this low-energy range.

The right matrix represents the posterior correlation matrix obtained with the BFMC method, using one single PIE measurement as the $E$ value, namely the isotopic concentration of ${ }^{239} \mathrm{Pu} .{ }^{239} \mathrm{Pu}$ was not present in the fuel at the beginning of the sample irradiation, but it is builtup from ${ }^{238} \mathrm{U}$ during the irradiation cycles. The longer the irradiation, the more ${ }^{239} \mathrm{Pu}$ is produced by neutron capture on ${ }^{238} \mathrm{U}$, but it also disappears because of its own fission and capture cross sections. Therefore, the remaining concentration of ${ }^{239} \mathrm{Pu}$ after irradiation (and cooling time) is increased by the ${ }^{238} \mathrm{U}(\mathrm{n}, \gamma)$ reactions, and 

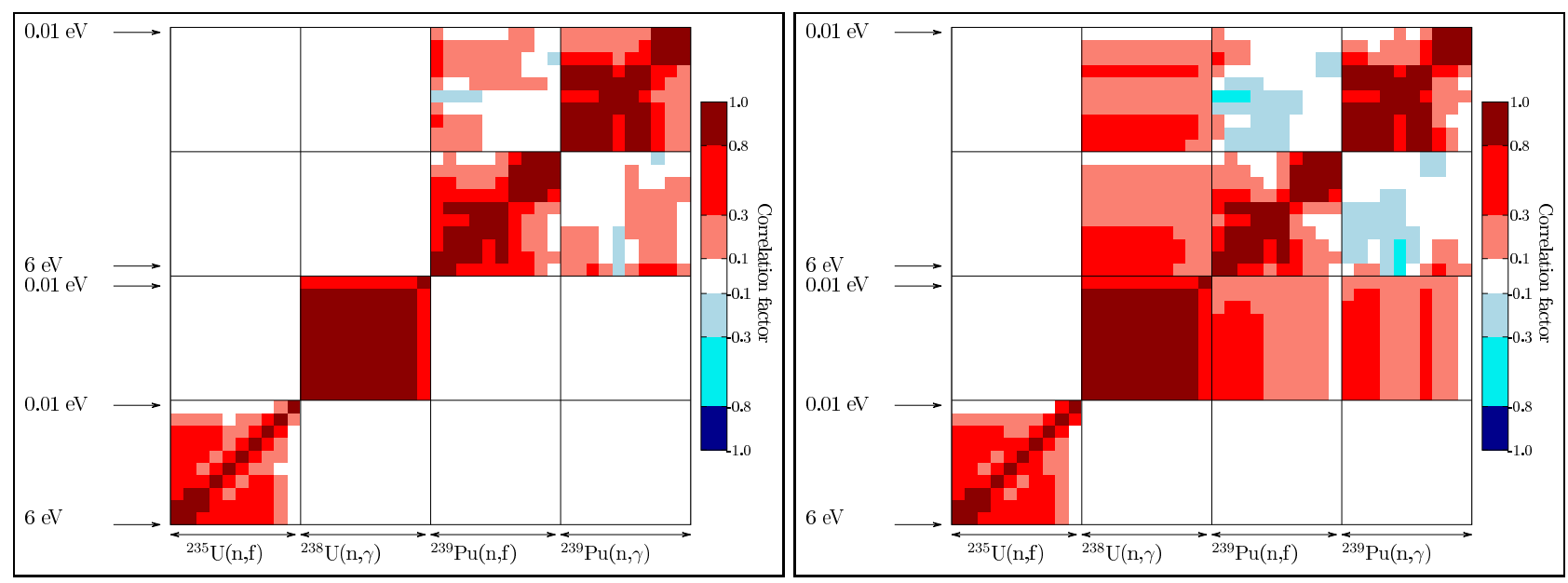

Fig. 2. Case of correlations between various actinide cross sections. Left: prior correlation matrix without PIE data; right: posterior correlation matrix using the PIE measurement from ${ }^{239} \mathrm{Pu}$.

also decreased by the two main ${ }^{239} \mathrm{Pu}$ reactions in this energy range. This case can typically be understood with the simplified definition of the correlation presented in Section 3:

- ${ }^{239} \mathrm{Pu}$ disappears because of both ${ }^{239} \mathrm{Pu}$ capture and fission cross sections. Therefore, keeping the ${ }^{239} \mathrm{Pu}$ isotopic concentration constant implies negative correlation between ${ }^{239} \mathrm{Pu}(\mathrm{n}, \mathrm{f})$ and ${ }^{239} \mathrm{Pu}(\mathrm{n}, \gamma)$.

- ${ }^{239} \mathrm{Pu}$ is created by ${ }^{238} \mathrm{U}(\mathrm{n}, \gamma)$ and disappears because of both ${ }^{239} \mathrm{Pu}$ capture and fission cross sections. Therefore, keeping the ${ }^{239} \mathrm{Pu}$ isotopic concentration constant implies a positive correlation between ${ }^{238} \mathrm{U}(\mathrm{n}, \gamma)$ and ${ }^{239} \mathrm{Pu}$ cross sections.

These simple explanations allow to understand the correlations appearing between ${ }^{238} \mathrm{U}$ and ${ }^{239} \mathrm{Pu}$ in the case of the right matrix of Figure 2. As in the case of $\mathrm{Cm}$ and $\mathrm{Pu}$ capture cross sections, it is important to keep in mind the choice of the experimental value $E$, used to calculate the weighted posterior correlations.

\subsection{Example 3: Correlations among fission yields}

The next example concerns the correlations between fission yields of different actinides, and does not involve cross sections. During the irradiation of the GU1 sample, ${ }^{235} \mathrm{U}$ is being burned mainly due to its fission cross section, and at the same time, the plutonium isotopes are being built-up. Two of them are also contributing to the fission rates: ${ }^{239} \mathrm{Pu}$ and ${ }^{241} \mathrm{Pu}$, as their thermal fission cross sections are relatively large (at the end of irradiation, the integral fission rates are $44 \%, 8 \%, 38 \%$ and $10 \%$ for ${ }^{235} \mathrm{U}$, ${ }^{238} \mathrm{U},{ }^{239} \mathrm{Pu}$ and ${ }^{241} \mathrm{Pu}$, respectively). Therefore, the three isotopes with an important thermal fission cross section, as well as their fission yields contribute to the creation of fission products. One can expect that by considering the measured concentration $E$ from a given fission product, correlations between the fission yields from these three isotopes should be observed. This is the case presented in Figure 3 where $E$ is the measured concentration for ${ }^{133} \mathrm{Cs}$.
Again, the left matrix is the prior correlation matrix. Only a limited number of fission yields is presented here, 10 for each actinide. As observed, the prior matrix contains a very limited number of correlations, being a common feature of all evaluated nuclear data libraries. The prior correlations are due to the in-house tool, as explained in the introduction. The right matrix presents the posterior correlations obtained by using the measured isotopic composition of ${ }^{133} \mathrm{Cs}$ as $E$ to calculate the weighted posterior distribution. This fission product is produced by the three mentioned actinides, with different contributions. It is nevertheless produced indirectly, as for mass 133, the main isotope directly produced by fission is ${ }^{133} \mathrm{I}$ (with the highest fission yield). This iodine isotope, having a short half-life of about 8 days, decays to ${ }^{133} \mathrm{Cs}$ during the irradiation and cooling times. Therefore three fission reactions lead to the creation of this isotope, via ${ }^{133} \mathrm{I}$. In order to keep it constant, the ${ }^{133}$ I fission yields for the three main fissioning actinides are negatively correlated, as observed in Figure 3. It is interesting to note that a very strong negative correlation appears between the ${ }^{133} \mathrm{I}$ fission yields of ${ }^{235} \mathrm{U}$ and ${ }^{239} \mathrm{Pu}$, since they are the two main contributors to mass 133 by fission.

\subsection{Example 4: Correlations between fission yields and cross sections}

The example presented in this section is representative of a number of cases. Similar correlations between fission yields and capture cross sections were also observed considering the isotopic compositions of ${ }^{145} \mathrm{Nd},{ }^{154} \mathrm{Sm}$, or ${ }^{134} \mathrm{Cs}$.

As mentioned, the production of a measured fission product can depend on many fission yields. These fission products can either be directly the isotope of interest, or they can be isotopes subsequently decaying to the observed isotopes. Moreover, neutron capture cross sections can also contribute to the decrease of the target nucleus population (as well as to the increase of the 

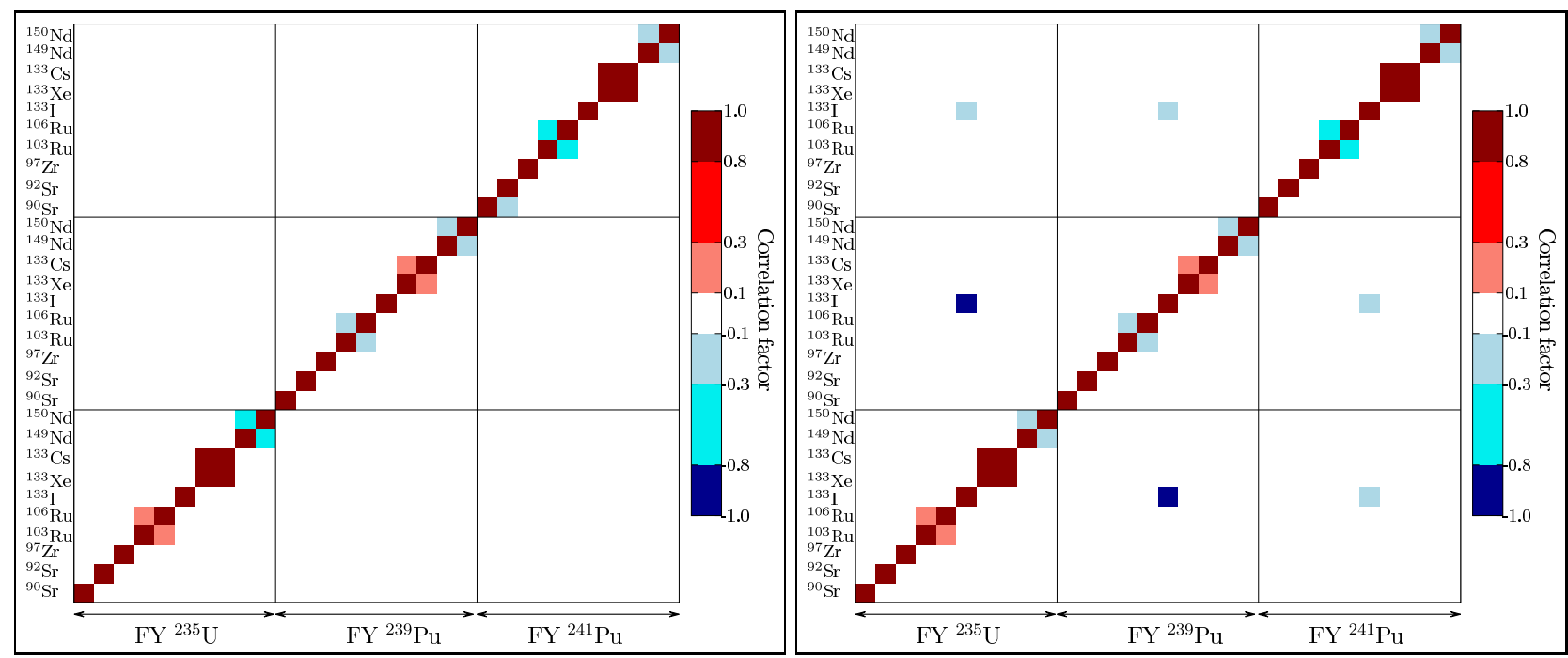

Fig. 3. Case of correlations between fission yields from ${ }^{235} \mathrm{U},{ }^{239} \mathrm{Pu}$ and ${ }^{241} \mathrm{Pu}$. Left: prior correlation matrix without PIE data; right: posterior correlation matrix using the PIE measurement from ${ }^{133} \mathrm{Cs}$.
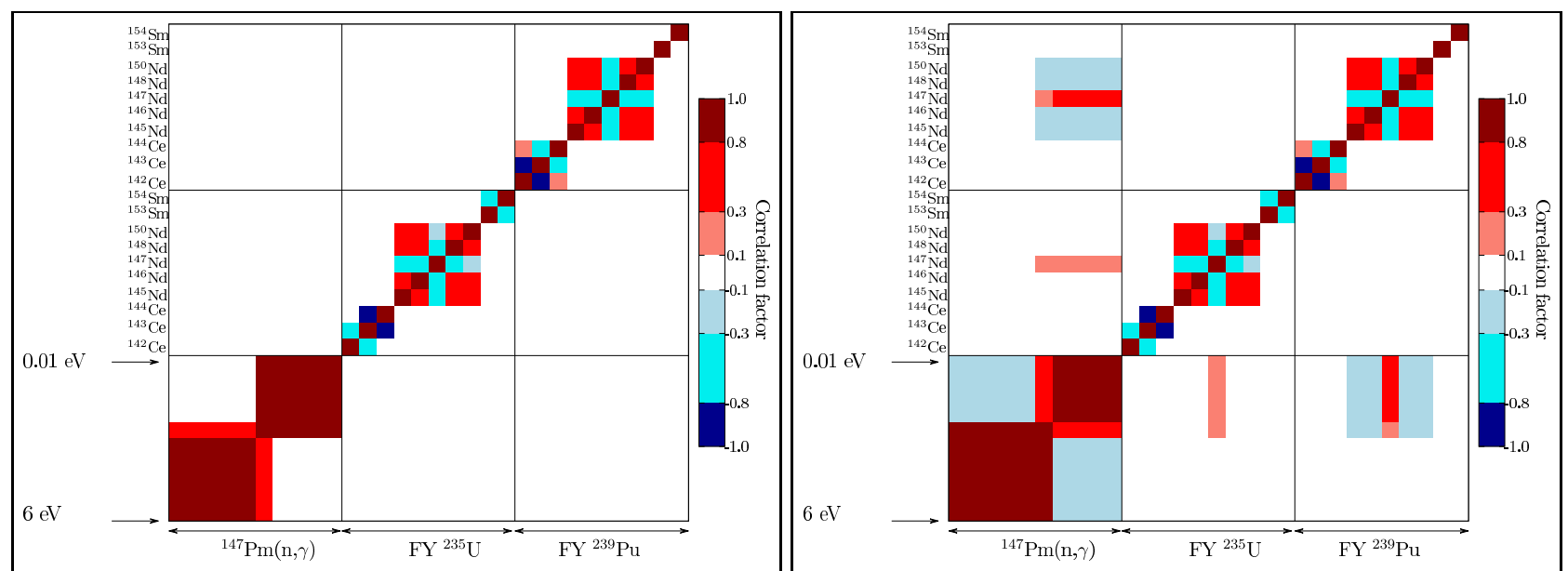

Fig. 4. Case of correlations between ${ }^{147} \mathrm{Pm}(\mathrm{n}, \gamma)$ and fission yields from ${ }^{235} \mathrm{U}$ and ${ }^{239} \mathrm{Pu}$. Left: prior correlation matrix without PIE data; right: posterior correlation matrix using the PIE measurement from ${ }^{147} \mathrm{Sm}$.

daughter nucleus population). In this case, positive correlations are expected between specific fission yields and the capture cross section(s) which depopulate them, as explained in the example of Section 3. The example presented in Figure 4 illustrates such effects. It represents the case of correlations between specific fission yields and a capture cross section. Again, the left figure is the prior correlation matrix, between the ${ }^{147} \mathrm{Pm}(\mathrm{n}, \gamma)$ cross section and a selection of fission yields of ${ }^{235} \mathrm{U}$ and ${ }^{239} \mathrm{Pu}$. As observed before, very limited correlations among fission yields exist, except for the $\mathrm{Nd}$ isotopes, presenting a negative correlation between ${ }^{147} \mathrm{Nd}$ and other $\mathrm{Nd}$ isotopes.

The right correlation matrix is obtained from the posterior distribution computed using the measured isotopic concentration of the ${ }^{147} \mathrm{Sm}$ fission product as the experimental constraint $E$. As in the previous example, it is not directly produced by fission. The short-lived ${ }^{147} \mathrm{Nd}$ (half-life of 11 days) is first produced, decaying to ${ }^{147} \mathrm{Pm}$ (half-life of 2.6 years) by beta emission, which finally produces ${ }^{147} \mathrm{Sm}$ also by beta decay. Because the halflife of ${ }^{147} \mathrm{Nd}$ is short compared to the irradiation time, its capture rate is small compared to the one of ${ }^{147} \mathrm{Pm}$. Additionally, ${ }^{147} \mathrm{Sm}$ does not disappear much because of its own capture cross section, as it is only slowly produced by decay over the irradiation and cooling periods (each being 3.5 and 3 years). In the present case, the ${ }^{147} \mathrm{Pm}(\mathrm{n}, \gamma)$ cross section and the ${ }^{147} \mathrm{Nd}$ fission yields are removing and creating ${ }^{147} \mathrm{Sm}$, respectively. This explains the observed positive correlations between the ${ }^{147} \mathrm{Pm}(\mathrm{n}, \gamma)$ cross section and ${ }^{147} \mathrm{Nd}$ fission yields for ${ }^{235} \mathrm{U}$ and ${ }^{239} \mathrm{Pu}$ on Figure 4. Additionally, because ${ }^{147} \mathrm{Nd}$ is negatively correlated with the other $\mathrm{Nd}$ isotopes in the prior matrix, variations of ${ }^{147} \mathrm{Nd}$ are also in opposite directions with the other $\mathrm{Nd}$ isotopes in the posterior matrix, although the negative correlation is weaker than in the prior case.

This example illustrates that correlations between XS and FY can be built, depending on the specific analyzed case. As mentioned, this example is not unique, and other 
ones were found. It is nevertheless the first time that these correlations are highlighted and even quantified. From the point of view of the evaluation of nuclear data, it is an additional evidence that it is worthwhile to consider correlations between different types of quantities across various isotopes: cross sections, $\nu$ (number of emitted neutron per fission), $\chi$ (energy spectra of these neutrons), fission yields, but also single and double differential spectra. For the time being, only correlations from theoretical reaction calculations and from the analysis of differential data are explicitly included in general purpose nuclear data libraries.

\section{Discussion}

The above examples illustrate that it is possible to generate correlations between virtually all the nuclear data that contribute to a given measured experimental value $E$. Criticality benchmarks, which have traditionally been used to select candidates for inclusion in evaluated nuclear data libraries, have been shown to induce correlations between cross sections, fission neutrons (both nubar and prompt fission neutron spectra), across energies and isotopes [26]. Those correlations are already present in the central values of evaluated nuclear data libraries since the "right" combination of cross sections, fission neutron observables were carefully selected (within the evaluated uncertainties) to optimize agreement with a large array of criticality benchmarks. The ENDF/B-VIII.0 library [1] acknowledges the calibration of the nubar observable for major actinides in order to "optimize $\mathrm{k}_{\mathrm{eff}}$ criticality" and so does the JEFF3.3 library [2]. So far the correlations stemming from use of integral constraints are only reflected in the central values, and not in the covariance matrices, leading to the apparent paradox of those libraries allowing good accurate (within 1 or $2 \sigma$ of the experiment) calculation of experimental values, while producing propagated uncertainties that are significantly larger. That issue was discussed at length in reference [27] but, at the time, it was not settled.

The examples shown in the present paper help clarifying this debate. In our example 1, correlations between the ${ }^{242} \mathrm{Pu}(\mathrm{n}, \gamma)$ and ${ }^{244} \mathrm{Cm}(\mathrm{n}, \gamma)$ cross-sections, are of opposite directions depending on which measured isotope $E\left({ }^{244} \mathrm{Cm}\right.$ or $\left.{ }^{245} \mathrm{Cm}\right)$ was used for the calculation the weighted posterior. It is obvious that those correlations are not universal enough to be included in a general purpose evaluated nuclear data library, and belong to adjusted application libraries. Similarly, example 3, which exhibits strong negative correlations between the ${ }^{133} \mathrm{I}$ fission yields of three actinides when the measured ${ }^{133} \mathrm{Cs}$ production is used to calculate the posterior, is only relevant if ${ }^{133} \mathrm{Cs}$ is measured. Therefore such correlations do not belong to a general purpose evaluated nuclear data library. Example 4 shows that the use of integral experimental constraints in the calculation of a posterior distribution $\left({ }^{147} \mathrm{Sm}\right.$ in this case) can even generate correlations between capture cross-sections and fission yields. Such correlations also do not belong to a general purpose evaluated nuclear data library.
On the other hand, those correlations are essential in order to account for the measured quantities with the precision needed by a number of applications. They can help in reducing calculated uncertainties on integral quantities, without necessarily changing the uncertainties on the relevant differential quantities. One of the underlying assumptions is that all parameters of the integral experiments are enough under control for not affecting the posterior quantities. Consequently, such correlations need to find their place in adjusted application libraries. Conversely, for the same reason, general purpose libraries should not be expected to be fully usable in applications without some adjustment (and inclusion of the needed correlations).

The path forward from there lies in the careful organization of the interface between the general purpose and the adjusted applications libraries. Future general purpose evaluated nuclear data libraries should make sure that they rely on as little integral information as possible during their evaluation (e.g. by including FY correlations based on differential measurements), and explicitly quantify the effect of this integral information in the associated covariance files. Additionally, automatic mechanisms (algorithms and codes systems) for nuclear data adjustment should be associated to evaluated nuclear data libraries, so that adjusted libraries can be easily generated and in a reproducible way. The BFMC, BMC, MOCABA methods [6-9] as well as the other methods presented in references [10-20] all qualify for such a goal to some extent (a similar new paradigm is suggested by [46]). If automated adjustment of libraries becomes easily achievable and a quantification of the "distance" between two nuclear data files (such as the overlap between the multivariate Gaussian distributions representing the libraries and their associated covariance matrices) is implemented, then a possible quality criterion for general purpose nuclear data libraries could be that the distance between the general purpose library and the multiple derived adjusted applications libraries is minimum.

\section{Conclusion}

This paper highlights cases of correlations between cross sections and fission yields due to selected integral measurements. Specifically, the use of the measured isotopic compositions from an irradiated sample with the BFMC method leads to nuclear data correlations not previously observed. It is an additional example that various types of nuclear data can be correlated together by the use of integral experimental constraints. If taken into account in specific evaluated libraries, such correlations can improve calculations of integral quantities and answer requests from a number of users, wishing for smaller calculated uncertainties. Because such correlations are constructed with specific measurements, and are case dependent, it is advocated that such correlations (and adjusted nuclear data) find their place in dedicated adjusted libraries. This possibility can improve the user's satisfaction, but also emphasizes the fact that current nuclear data evaluations do not lead to a unique set of cross sections, nubar or 
fission yields. These sets should be validated on a case by case basis, being very careful to clearly define their domain of validation and exercising extreme caution when crossing the boundaries of these domains. In the end, the quantitative agreement between the experimental values and the simulated ones, on both the central values and uncertainties constitutes a strong validation of the nuclear data used in simulations. For the uncertainties, such comparisons constitute the only nonintrusive ${ }^{1}$ way to validate an existing evaluated covariance file [47].

The work of D. Rochman was partly supported by swissnuclear, the association of the Swiss nuclear power station operators, with the COLOSS project. It was also partly funded by the European Union's Horizon 2020 Research and Innovation Programme under grant agreement No 847593.

\section{Author contribution statement}

Both authors defined the necessary steps to make correlations visible between fission yields and cross sections. D. Rochman performed the various calculations. E. Bauge and D. Rochman performed the analysis of the results. The paper was written by both co-authors.

\section{References}

1. D.A. Brown et al., ENDF/B-VIII.0: the 8th major release of the nuclear reaction data library with CIELO-project cross sections, new standards and thermal scattering data, Nucl. Data Sheets 148, 1 (2018)

2. A.J. Plompen et al., The joint evaluated fission and fusion nuclear data library, JEFF-3.3, Eur. Phys. J. A 56, 181 (2020)

3. K. Shibata et al., JENDL-4.0: a new library for nuclear science and engineering, J. Nucl. Sci. Technol. 48, 1 (2011)

4. A. Koning, D. Rochman, J. Sublet, N. Dzysiuk, M. Fleming, S. van der Marck, TENDL: complete nuclear data library for innovative nuclear science and technology, Nucl. Data Sheets 155, 1 (2019)

5. A.D. Carlson et al., Evaluation of the neutron data standards, Nucl. Data Sheets 148, 143 (2018)

6. E. Bauge, P. Dossantos-Uzarralde, Evaluation of the covariance matrix of ${ }^{239} \mathrm{Pu}$ neutronic cross sections in the continuum using the backward-forward Monte-Carlo method, J. Korean Phys. Soc. 59, 1218 (2011)

7. A.J. Koning, Bayesian Monte Carlo method for nuclear data evaluation, Eur. Phys. J. A 51, 184 (2015)

8. A. Hoefer, O. Buss, M. Hennebach, M. Schmid, D. Porsch, MOCABA: a general Monte Carlo-Bayes procedure for improved predictions of integral functions of nuclear data, Ann. Nucl. Energy 77, 514 (2015)

9. E. Bauge, Full model nuclear data and covariance evaluation process using TALYS, Total Monte Carlo and backward-forward Monte Carlo, Nucl. Data Sheets 123, $201(2015)$

\footnotetext{
${ }^{1}$ In this context, nonintrusive means that only the evaluated covariance data file is being examined for validation, without relying on knowledge of the methods and data used to generate it.
}

10. S. Pelloni, D. Rochman, Resonance parameter adjustment in the resolved region based upon an Asymptotic Generalized Linear Least-Squares methodology in conjunction with the Monte Carlo method, Ann. Nucl. Eng. 145, 107509 (2020)

11. C. de Saint Jean, P. Archier, E. Privas, G. Noguère, On the use of Bayesian Monte-Carlo in evaluation of nuclear data, EPJ Web Conf. 146, 02007 (2017)

12. S. Pelloni, Comparison of progressive incremental adjustment sequences for cross-section and variance/covariance data adjustment by analyzing fast-spectrum systems, Ann. Nucl. Energy 106, 33 (2017)

13. S. Pelloni, D. Rochman, Cross-section adjustment in the fast energy range on the basis of an Asymptotic Progressing nuclear data Incremental Adjustment (APIA) methodology, Ann. Nucl. Energy 115, 323 (2018)

14. M. Salvatores et al., Methods and issues for the combined use of integral experiments and covariance data: results of a NEA International Collaborative Study, Nucl. Data Sheets 118, 38 (2014)

15. C. de Saint Jean, P. Archier, E. Privas, G. Noguère, O. Litaize, P. Leconte, Evaluation of cross section uncertainties using physical constraints: focus on integral experiments, Nucl. Data Sheets 123, 178 (2015)

16. R. Capote, D.L. Smith, A. Trkov, M. Meghzifene, A new formulation of the Unified Monte Carlo Approach (UMCB) and cross-section evaluation for the dosimetry reaction ${ }^{55} \mathrm{Mn}(\mathrm{n}, \gamma){ }^{56} \mathrm{Mn}$, J. ASTM Int. 9, 1 (2012)

17. D.L. Smith, A unified Monte Carlo approach to fast neutron cross section data evaluation, in Proceedings of the $8^{\text {th }}$ International Topical Meeting on Nuclear Applications and Utilization of Accelerators, Pocatello, July 29-August 2 (2007), p. 736

18. P. Helgesson, H. Sjöstrand, A.J. Koning, J. Ryden, D. Rochman, E. Alhassan, S. Pomp, Combining total Monte Carlo and Unified Monte Carlo: Bayesian nuclear data uncertainty quantification from auto-generated experimental covariances, Progr. Nuclear Energy 96, 76 (2017)

19. T. Watanabe, T. Endo, A. Yamamoto, Y. Kodama, Y. Ohoka, T. Ushio, Cross section adjustment method based on random sampling technique, J. Nucl. Sci. Technol. 51, $590(2014)$

20. T. Kawano, K.M. Hanson, S. Frankle, P. Talou, M.B. Chadwick, R.C. Little, Evaluation and propagation of the $239 \mathrm{Pu}$ fission cross-section uncertainties using a Monte Carlo technique, Nucl. Sci. Eng. 153, 1 (2006)

21. C. De Saint Jean, P. Archier, E. Privas, G. Noguère, B. Habert, P. Tamagno, Evaluation of neutron-induced cross sections and their related covariances with physical constraints, Nucl. Data Sheets 148, 383 (2018)

22. D. Siefman, M. Hursin, H. Sjostrand, G. Schnabel, D. Rochman, A. Pautz, Data assimilation of post-irradiation examination data for fission yields from GEF, Eur. Phys. J. N 6, 52 (2020)

23. E. Alhassan, D. Rochman, H. Sjostrand, A. Vasiliev, A.J. Koning, H. Ferroukhi, Bayesian updating for data adjustments and multi-level uncertainty propagation within Total Monte Carlo, Ann. Nucl. Eng. 139, 107239 (2020)

24. D. Rochman, A. Vasiliev, H. Ferroukhi, S. Pelloni, E. Bauge, A.J. Koning, Correlation nu-sigma for U-Pu in the thermal and resonance neutron range via integral information, Eur. Phys. J. Plus 134, 453 (2019) 
25. D. Siefman, M. Hursin, D. Rochman, S. Pelloni, A. Pautz, Stochastic vs. sensitivity-based integral parameter and nuclear data adjustments, Eur. Phys. J. Plus 133, 429 (2018)

26. D. Rochman, E. Bauge, A. Vasiliev, H. Ferroukhi, S. Pelloni, A.J. Koning, J.Ch. Sublet, Monte Carlo nuclear data adjustment via integral information, Eur. Phys. J. Plus 133, 537 (2018)

27. E. Bauge, D. Rochman, Cross-observables and crossisotopes correlations in nuclear data from integral constraints, Eur. Phys. J. N 4, 35 (2018)

28. D. Rochman, E. Bauge, A. Vasiliev, H. Ferroukhi, G. Perret, Nuclear data correlation between different isotopes via integral information, Eur. Phys. J. N 4, 7 (2018)

29. D. Rochman, E. Bauge, A. Vasiliev, H. Ferroukhi, Correlation nu-sigma-chi in the fast neutron range via integral information, Eur. Phys. J. N 3, 14 (2017)

30. J.Ch. Sublet et al., Neutron-induced damage simulations: beyond defect production cross-section, displacement per atom and iron-based metrics, Eur. Phys. J. Plus 134, 350 (2019)

31. O. Leray, L. Fiorito, D. Rochman, H. Ferroukhi, A. Stankovskiy, G. Van den Eynde, Uncertainty propagation of fission product yields to nuclide composition and decay heat for a PWR $\mathrm{UO}_{2}$ fuel assembly, Progr. Nucl. Energy 101, 486 (2017)

32. D. Rochman et al., Nuclear data uncertainties for typical LWR fuel assemblies and a simple reactor core, Nucl. Data Sheets 139, 1 (2017)

33. R.W. Mills, Uncertainty propagation of fission product yield data in spent fuel inventory calculations, Nucl. Data Sheets 118, 484 (2014)

34. T. Frosio, T. Bonaccorsi, P. Blaise, Fission yields and cross section uncertainty propagation in Boltzmann/Bateman coupled problems: Global and local parameters analysis with a focus on MTR, Ann. Nucl. Eng. 98, 43 (2016)

35. J.B. Briggs, J.D. Bess, J. Gulliford, Integral benchmark data for nuclear data testing through the ICSBEP \& IRPhEP, Nucl. Data Sheets 118, 396 (2014)

36. J.B. Briggs, J. Gulliford, An overview of the international reactor physics experiment evaluation project, Nucl. Sci. Eng. 178, 269 (2014)

37. I. Kodeli, E. Sartori, B. Kirk, SINBAD shielding benchmark experiments status and planned activities, in
Proceedings of the American Nuclear Society, 14th Biennial Topical Meeting of the Radiation Protection and Shielding Division, Carlsbad New Mexico, USA, April 3-6, 2006

38. M. Tardy, S. Kitsos, G. Grassi, A. Santamarina, L. San Felice, C. Riffard, First burnup credit application including actinides and fission products for transport and storage cask by using French experiments, J. Nucl. Sci. Technol. 52, 1008 (2015)

39. D. Rochman, A. Vasiliev, H. Ferroukhi, M. Seidl, J. Basualdo, Improvement of PIE analysis with a full core simulation: the U1 case, Ann. Nucl. Eng. 148, 107706 (2020)

40. D. Rochman, A. Vasiliev, H. Ferroukhi, M. Pecchia, Consistent criticality and radiation studies of Swiss spent nuclear fuel: the $\mathrm{CS}_{2} \mathrm{M}$ approach, J. Hazard. Mater. 357, 384 (2018)

41. ARIANE International Programme Final Report, Belgonucléaire, AR2000/15 BN Ref. 0000253/221, Revision B, December 2000

42. F. Michel-Sendis et al., SFCOMPO-2.0: an OECD NEA database of spent nuclear fuel isotopic assays, reactor design specifications, and operating data, Ann. Nucl. Eng. 110, 779 (2017)

43. D. Rochman, A. Vasiliev, H. Ferroukhi, M. Hursin, Analysis for the ARIANE GU1 sample: isotopic compositions and decay heat, Ann. Nucl. Eng. (2021), in press

44. J. Rhodes, K. Smith, D. Lee, CASMO-5 development and applications, in Proceedings of the PHYSOR-2006 conference, ANS Topical Meeting on Reactor Physics, Vancouver, BC, Canada, September 10-14, Vancouver, BC, Canada, September 10-14 (2006), p. B144.

45. O. Leray, D. Rochman, P. Grimm, H. Ferroukhi, A. Vasiliev, M. Hursin, G. Perret, A. Pautz, Nuclear data uncertainty propagation on spent fuel nuclide compositions, Ann. Nucl. Eng. 94, 603 (2016)

46. M. Herman, D.A. Brown, M.B. Chadwick, W. Haeck, T. Kawano, D. Neudecker, P. Talou, A. Trkov, M.C. White, New paradigm for nuclear data evaluation, in Proceedings of the International Conference on Nuclear Data for Science and Technology, Beijing, China, May 19-24, 2019, EPJ Web Conf. 239, 11001 (2020)

47. E. Bauge et al., Coherent investigation of nuclear data at CEA DAM: Theoretical models, experiments and evaluated data, Eur. Phys. J. A 48, 113 (2012)

Cite this article as: Dimitri Alexandre Rochman and Eric Bauge, Fission yields and cross sections: correlated or not?, EPJ Nuclear Sci. Technol. 7, 5 (2021) 